\title{
Criminologie
}

\section{Une expérience pilote de sentence de travaux communautaires}

\section{Julien Fortier et Gérald Gallant}

Volume 12, numéro 2, 1979

Probation : aide ou contrainte?

URI : https://id.erudit.org/iderudit/017106ar

DOI : https://doi.org/10.7202/017106ar

Aller au sommaire du numéro

Éditeur(s)

Les Presses de l'Université de Montréal

ISSN

0316-0041 (imprimé)

1492-1367 (numérique)

Découvrir la revue

Citer cet article

Fortier, J. \& Gallant, G. (1979). Une expérience pilote de sentence de travaux communautaires. Criminologie, 12(2), 24-40. https://doi.org/10.7202/017106ar d'utilisation que vous pouvez consulter en ligne.

https://apropos.erudit.org/fr/usagers/politique-dutilisation/ 
Plusieurs pays ont récemment expérimenté les différentes façons d'utiliser le travail communautaire comme une alternative aux mesures pénales traditionnelles. En Angleterre (1970, 1973, 1974), le comité consultatif sur le système pénal lançait pour la première fois l'idée de sentence de travaux communautaires. En 1973, une expérience pilote était mise sur pied, suite à laquelle l'application du concept s'étendait à toute l'Angleterre et au pays de Galles. Aux États-Unis (1977), la sentence de travaux comunautaires est mise à l'essai en Oregon en 1972. Au Canada (1976), quelques expériences dans ce domaine ont été menées depuis 1974 de façon officielle et officieuse en Colombie-Britannique, en Ontario et en NouvelleÉcosse.

En 1976, le service de Probation du ministère de la Justice du Québec se donnait comme objectif de procéder à la création de mécanismes permettant l'application de la sentence de travaux communautaires dans les districts judiciaires du Québec. Plusieurs raisons motivaient cette décision. D'une part, la nécessité de développer des mesures alternatives à l'incarcération avait été soulignée par certains juges et certains agents de probation. De plus, bien que quelques expériences éparses de sentences de travaux communautaires avaient été tentées au Québec, aucune structure officielle ne pouvait garantir leur application. Cependant, avant d'implanter un tel programme dans tout le Québec, il apparaissait essentiel de mettre sur pied une expérience pilote afin d'examiner jusqu'à quel point la sentence de travaux communautaires correspondait à un besoin pour les tribunaux et ses usagers et d'évaluer dans quelle mesure et dans quelles conditions le concept était applicable dans un contexte québécois. Six régions pilotes furent donc sélectionnées : deux de type urbain, soit Hull et Longueuil, deux de type semi-

* Les auteurs :

- Julien Fortier (B.Sc.), agent de probation, Valleyfield, P.Q.

la Probation, Québec, P.Q.

- Gérald Gallant (M.), adjoint aux projets spéciaux, direction de 
urbain, soit St-Hyacinthe et Valleyfield et deux régions rurales, soit Montmagny et Val-d'Or.

Tel que conçu initialement, le travail commuautaire par les contrevenants doit être envisagé comme une nouvelle alternative à la peine carcérale dans le cas où l'intérêt public ne la nécessite pas. Il permet à l'accusé de devenir un élément social conscient de ses responsabilités face à la communauté en réparant le tort qu'il lui a causé. L'originalité du programme repose dans le fait qu'il offre au justiciable, par l'entremise du processus légal, l'opportunité de purger une sentence hors du contexte carcéral par l'accomplissement de travail bénévole auprès d'organismes à but non lucratif ceuvrant pour la communauté.

Nous allons traiter d'abord du cadre d'application de la mesure de sentence de travaux communautaires, tel qu'il a été conçu initialement et des mécanismes qui ont permis d'évaluer l'expérience pilote. Dans un deuxième temps, nous allons décrire comment cette structure d'application a été mise à l'épreuve au cours du déroulement et quelles ont été les modifications d'ordre majeur à apporter. L'analyse de trois cas types de sentence de travaux communautaires sera présentée finalement dans le but de mieux illustrer les différentes dimensions inhérentes à la mise en application de cette mesure.

\section{THÉORIE ET POLITIQUE D'APPLICATION}

Les objectifs du programme s'énoncent comme suit : * fournir au tribunal et à ses usagers une mesure alternative aux peines d'incarcération dans les cas où l'intérêt public n'est pas mis en danger, permettre au justiciable de réparer par le moyen d'une sentence socialement productive le tort criminellement causé et donner à la communauté l'occasion de participer de façon active à l'administration de la justice. .

Dans le cadre légal actuel, une sentence de travaux communautaires doit faire l'objet d'une condition spécifique d'une ordonnance de probation en vertu des alinéas 663(1)a) et 663 (2)h) du Code criminel. Advenant que des changements soient souhaitables au cours du déroulement d'une sentence de travaux communautaires, ou encore que le justiciable fasse preuve de mauvaise volonté en ne se conformant pas à une telle ordonnance, les articles du Code criminel relatifs à l'entrée en vigueur de 
l'ordonnance, à ses modifications et au défaut de se conformer s'appliquent mutatis mutandis à une ordonnance comportant une condition spécifique de travaux communautaires.

Concrètement, la préparation d'une sentence de travaux communautaires se déroule comme suit. D'abord, toute imposition d'une telle mesure doit être nécessairement précédée d'un rapport présentenciel. Une demande de sentence de travaux communautaires peut provenir de deux sources. D'une part, la cour peut, avant le prononcé de la sentence, demander au service de Probation d'étudier la possibilité de l'octroi d'une sentence de travaux communautaires. D'autre part, il se peut que la cour fasse la demande d'un rapport présentenciel sans préciser pour autant l'examen de cette possibilité. L'agent de probation peut alors conclure dans son rapport que cette mesure convient le mieux à l'accusé.

Dans les deux cas, l'agent de probation examine le candidat en fonction de certains critères précis. Tout d'abord, sous réserve de circonstances particulières justifiant son octroi, un tel type de sentence ne peut être accordé à un alcoolique chronique, à un usager régulier de drogue majeure, à un individu présentant des problèmes de personnalité marqués, à un individu fortement impliqué dans une carrière criminelle et d'une façon plus générale qui ne consent pas à se soumettre à une telle mesure. De plus, une attention particulière est accordée à ses aptitudes personnelles de travail, à son attitude face à une telle sentence et à la gravité objective du délit criminel en cause. Le rapport présentenciel concluant, ou non, à l'opportunité de ce type de sentence est ensuite acheminé à la cour. Si celle-ci abonde dans le sens de la recommandation, le juge donne son accord de principe à l'agent de probation et reporte le prononcé de la sentence à quelques semaines. Durant cette période, l'agent de probation identifie une ressource communautaire qu'il choisit en fonction des aptitudes du candidat, de sa disponibilité, du délit impliqué et du nombre d'heures convenu avec la cour lors de l'accord de principe donné par le juge.

La ressource communautaire est également sélectionnée en fonction des critères d'admissibilité précis : seuls les organismes à but non lucratif sont éligibles. De plus, ces ressources doivent être dotées de police d'assurance contre le feu, le vol et la responsabilité civile. Outre ces critères, la ressource communautaire 
a la responsabilité de vérifier que le nombre d'heures prescrit dans l'ordonnance soit effectué durant les délais prévus et elle doit faire rapport à l'agent de probation de tout manquement ou modification pouvant être apporté au plan de travail initial.

Dès que la ressource communautaire est choisie et qu'elle accepte de s'occuper du cas, une rencontre est organisée sur les lieux du travail en présence du justiciable, du superviseur communautaire et de l'agent de probation. Elle a pour but de présenter la ressource communautaire au candidat, de préciser les rôles de chacun au niveau du fonctionnement et du contrôle de la sentence, d'établir un horaire précis de travail et d'obtenir un accord définitif de toutes les parties impliquées. Tous ces renseignements sont ensuite remis à la cour sous forme d'un bref rapport complémentaire. Le tribunal prononce ensuite sa décision.

L'agent de probation pour sa part demeure en tout temps le premier responsable de l'application de la sentence. $\mathrm{nl}$ assure au cours du déroulement du processus le lien entre le justiciable, la ressource communautaire et la cour, puis il voit à ce que les travaux s'effectuent en conformité avec les modalités prévues et informe la cour de toute modification ou révocation à apporter concernant l'exécution de l'ordonnance.

La mise en application de cette expérience pilote s'est faite en deux phases, soit limplantation et le déroulement, et cela du ler avril 1977 au 31 mai 1978. Dès le début de l'expérience pilote, on a prévu des mécanismes de relevés de données qualitatives et quantitatives : réunions à intervalle régulier des responsables de chaque région pilote, formulaires d'évaluation destinés aux participants de l'expérience, étude de temps, formulaires de compilations statistiques, etc. Outre ces modalités utilisées au cours du déroulement de l'expérience, un questionnaire d'évaluation finale fut élaboré et administré, sous forme d'entrevues structurées, aux juges, aux procureurs, aux agents de probation, aux superviseurs des ressources communautaires et aux juticiables ayant participé à l'expérience.

Il est évident que les initiateurs du projet ont dû écarter au départ la possibilité d'évaluer jusqu'à quel point la mesure de sentence de travaux communautaires produisait l'évolution désirée des justiciables en termes de récidive ou de changement individuel. Le nombre de cas peu élevé et le manque d'infor- 
mation à long terme limitaient cette possibilité. Les préoccupations à court terme étaient de deux ordres : voir jusqu'à quel point la mesure de sentence de travaux communautaires correspond à un besoin et dans quelle mesure et dans quelles conditions le concept est applicable dans un contexte québécois.

Il convient maintenant d'examiner comment sur le plan empirique ont fonctionné les deux phases de ce projet, soit son implantation et son déroulement.

\section{L'IMPLANTATION ET LE DÉROULEMENT DE L'EXPÉRIENCE PILOTE}

\section{a) L'implantation du programme}

Au niveau des juges, l'implantation du programme a été effectuée sans trop de difficultés, si on tient compte du fait que l'introduction d'un tel type de programme dans le domaine de l'administration de la justice, surtout lorsqu'il est proposé comme une alternative à l'incarcération, ne peut s'effectuer sans une certaine résistance inhérente à tout changement. Cependant, plusieurs magistrats ont fait des commentaires concernant les assurances contre le feu, le vol et la responsabilité civile en affirmant qu'ils auraient souhaité que le ministère de la Justice élabore des dispositions légales relatives à cette question.

Au niveau des ressources communautaires, en plus du problème des assurances que plusieurs ont soulevé, on a posé des questions relatives au contexte légal dans lequel se déroulait une sentence de travaux communautaires et au rôle précis exigé de leur part.

Toutefois, comme la plupart des ressources communautaires étaient d'accord avec les objectifs d'un tel type de sentence, il n'y a eu aucun refus catégorique d'une éventuelle participation.

L'implication de ce projet pilote, sa préparation et son contrôle ont occasionné aux agents de probation une surcharge de travail due au fait que les effectifs n'ont pas été augmentés. Tout d'abord, ils avaient la responsabilité d'identifier, de sensibiliser et par la suite de recruter les ressources communautaires. De plus, ils devaient sensibiliser la cour et les procureurs à cette nouvelle mesure et répondre à leurs interrogations. Enfin, en raison de la nouveauté du programme, plusieurs ont éprouvé des difficultés à s'y adapter. 


\section{b) Déroulement du programme}

Rappelons que la préparation d'une sentence de travaux communautaires s'effectue en quatre étapes : l'origine de la demande, l'étude du candidat, l'approche d'une ressource communautaire et la recommandation de l'agent de probation au niveau du rapport présentenciel. Concernant l'origine de la demande, on a constaté à la suite de l'expérience pilote que sur un total de 72 cas suggérés pour l'étude, 62 ont été recommandés par les agents de probation lors de la rédaction du rapport présentenciel. Les 10 autres cas provenaient de la cour qui demandait au service de Probation de préparer un rapport présentenciel envisageant la possibilité d'une sentence de travaux communautaires.

Au niveau de l'évaluation des candidats, sur le total des 72 cas étudiés en fonction des critères d'admissibilité prévus au manuel des procédures, 50 cas ont fait l'objet d'une recommandation spécifique selon laquelle la sentence de travaux communautaires convenait le mieux à ces accusés. Les résultats de l'expérience pilote ont démontré la nécessité d'ajouter certains critères de sélection des candidats. Il s'est avéré, en effet, que les justiciables ayant des causes en suspens sont difficilement admissibles au programme du fait que dans certains cas, la cour a tout simplement refusé d'octroyer une sentence de travaux communautaires ou encore, que certains ont dû interrompre leurs travaux en raison d'une condamnation ultérieure à l'incarcération dans une autre cause. Aussi, lors du déroulement de l'expérience, on a constaté que les candidats ayant accompli avec succès leur sentence, avaient les caractéristiques particulières suivantes : une motivation à participer au programme, une certaine stabilité globale et une résidence à proximité du lieu de travail.

Pour faciliter l'approche auprès d'une ressource communautaire, il est apparu important, par ailleurs, de sensibiliser, préalablement à la mise en application du projet, une banque d'organismes à but non lucratif et d'identifier leurs objectifs et leurs caractéristiques particulières.

Au niveau du prononcé de la sentence, règle générale, la majorité des juges ont affirmé se sentir liés moralement par l'accord de principe donné, sauf pour les accusés qui commet- 
tent un nouveau délit durant la période se situant entre la date de cet accord et le prononcé de la sentence. Dans les cas où l'accord de principe n'a pas été respecté par la cour pour d'autres motifs, les justiciables concernés ont manifesté de l'amertume à l'égard de l'administration de la justice. Les agents de probation, pour leur part, ayant investi plusieurs heures de travail auprès des organismes communautaires, étaient hésitants à établir avec ces derniers de nouveaux contacts pour un autre cas.

Le fait de disposer du relevé des modalités d'application et de contrôle de la sentence, tel que contenu au rapport complémentaire, permet à la cour d'ordonner que l'accusé accomplisse un nombre d'heures déterminé de travail pour un organisme, et cela dans un délai prévu dans le cadre d'une ordonnance de probation.

Rappelons qu'au début de l'expérience, le comité chargé d'étudier les mécanismes d'application des sentences de travaux communautaires préconisait l'idée d'une nouvelle alternative à l'incarcération dans les cas où l'intérêt public ne nécessite pas une peine d'emprisonnement. Toutefois, l'évaluation démontre que la mesure de travaux communautaires a été ordonnée dans $60 \%$ des cas comme alternative stricte à la détention. Dans les autres cas, on a constaté que cette mesure a été utilisée bien que l'emprisonnement n'aurait pas été normalement nécessaire.

D'autre part, plusieurs juges ayant participé à l'expérience pilote ont cru bon de jumeler ce type de sentence à d'autres peines telles que l'amende, la restitution, la détention ou l'ordonnance de probation entraînant une surveillance. En effet, sur un total de 36 sentences de travaux communautaires, 29 ont été jumelées à une probation impliquant la surveillance, quatre à la restitution, deux à l'amende et une à la détention. Il n'en demeure pas moins que le Comité de sentence de travaux communautaires rejette le jumelage détention-travaux communautaires puisque l'objectif premier du programme est d'envisager cette mesure comme une alternative à l'incarcération. Le comité favorise toutefois le jumelage de la nouvelle mesure avec les autres types de sentence.

Nous allons traiter maintenant du contrôle de l'ordonnance de probation comportant des travaux communautaires au cours de l'expérience pilote. Trois acteurs principaux y sont 
impliqués : l'agent de probation, la ressource communautaire et le justiciable.

L'agent de probation doit vérifier si la formule d'ordonnance de travaux communautaires est conforme au procès-verbal de la sentence. De plus, au cours de l'accomplissement des travaux, il doit s'assurer que le justiciable se conforme au plan de travail élaboré et doit intervenir, au besoin, auprès de la ressource communautaire ou du justiciable pour suggérer, ou trouver une solution, à tout problème éventuel. Enfin, une fois les travaux terminés, l'agent de probation prépare un rapport d'évaluation en vue d'informer la cour des résultats obtenus.

Lors de l'expérience pilote, les ressources communautaires se sont acquittées de leurs responsabilités concernant les contrôles. Cependant, afin d'assurer un meilleur suivi du justiciable au cours du déroulement de la sentence, les résultats de l'évaluation démontrent qu'une seule personne à l'intérieur de l'organisme communautaire doit assumer la supervision.

Le rôle des justiciables assujettis à l'accomplissement de travaux communautaires consiste à effectuer le nombre d'heures de travail exigé par la cour dans les délais prescrits et selon l'horaire pré-établi. Au moment de la préparation du rapport d'évaluation de l'expérience pilote (1978), 25 sentences sur 37 étaient complétées. De ce total, $16 \%$ ont été qualifiées de succès théorique, en ce sens que les objectifs visés par les agents de probation ne furent que partiellement atteints, bien que les travaux furent exécutés en totalité dans les délais prescrits. En définitive, l'expérience menée démontre que le succès d'une telle mesure dépend, entre autres, de la précision avec laquelle le plan de travail a été élaboré lors de la préparation de la sentence, d'un contrôle étroit lors de son déroulement et de la compréhension de la philosophie de la nouvelle mesure par le justiciable. Au total, on a enregistré qu'un seul échec, signifiant par là que le sursitaire a refusé de compléter la totalité des travaux ordonnés par le juge.

\section{L'IMPACT CHEZ LE JUSTICIABLE}

En ce qui a trait à l'impact réel de cette mesure pénale au niveau du justiciable, il est encore trop tôt pour l'évaluer en termes de réhabilitation, de réinsertion sociale ou de récidive 
en raison du nombre restreint de cas et aussi à cause d'un manque de « follow-up > à long terme relatif aux indices de changements individuels. Cependant, une fois leur sentence de travaux communautaires terminée, on a constaté que plusieurs justiciables ont pu continuer d'assumer leurs responsabilités familiales, développer un peu plus de maturité, découvrir de nouvelles relations humaines et s'engager auprès de la communauté.

Dans le but de permettre une meilleure compréhension des différentes dimensions reliées à la préparation et à la mise en application de cette mesure, nous analyserons ici trois cas en particulier.

\section{a) Première histoire de cas}

En date du 28 janvier 1977, René... est déclaré coupable de quelques délits de fraude et la cour, avant de rendre sa sentence, demande au service de Probation de préparer un rapport présentenciel le concernant.

Lors de la première entrevue, l'agent de probation décèle chez René... les caractéristiques potentielles d'un candidat admissible à une sentence de travaux communautaires. Âgé de 32 ans, René... est marié et père de quatre enfants. Issu d'un milieu ouvrier relativement aisé, il a vécu son enfance et son adolescence sans présenter aucun problème de comportement majeur tout en réussissant assez bien sur le plan scolaire. Ses premières années de travail ont été valorisantes pour lui. Son emploi est rémunérateur, permanent et lui offre de bonnes conditions. Cependant, en raison d'une mauvaise administration de son budget, sa situation financière devient tellement critique qu'à un moment donné ses créanciers font saisir son salaire ce qui provoque son congédiement. Par la suite, durant plus de neuf ans, le sujet vit de prestations d'Aide sociale ne voulant pas travailler d'une façon permanente de crainte de faire saisir son salaire. Durant cette période d'oisiveté, il commet deux délits dont la possession d'alcool frelaté et le vol par effraction, en alléguant avoir besoin d'argent pour subvenir aux besoins de sa famille. Il invoque le même motif concernant son dernier délit pour lequel un rapport présentenciel a été demandé. Au plan des activités sociales, René... est très limité, mais il ne présente aucun problème d'alcoolisme ou d'usage de drogue. 
Après l'évaluation présentencielle, l'agent de probation indique dans son rapport qu'une sentence de travaux communautaires serait une mesure souhaitable pour cet individu. Les objectifs visés par cette mesure consistent à le rendre conscient de ses responsabilités face à la communauté, à le motiver éventuellement à se trouver un emploi régulier et à régler par les moyens légaux ses problèmes financiers.

La cour donne son accord de principe en établissant un quantum approximatif de 90 heures. L'agent de probation entreprend la recherche d'un organisme à but non lucratif situe a proximité de la résidence du justiciable. Des démarches sont ensuite entreprises auprès du directeur d'un centre de villégiature situé sur une île, desservant des mouvements de jeunes et d'adultes. Puis, il est convenu entre l'agent de probation et le directeur du centre que le travail prescrit consisterait dans l'entretien de l'île. Le justiciable ayant donné son consentement, l'agent de probation établit un horaire précis à la convenance de René... et du superviseur communautaire.

Lors de la comparution, la cour suspend le prononcé de la sentence pour une période d'un an et ordonne que René... accomplisse un travail communautaire selon les modalités établies par l'agent de probation.

La sentence se déroule sans aucun problème autant pour la ressource que pour le justiciable. Le sujet en plus d'effectuer ses tâches, met à la disposition du centre sa camionnette pour le transport des ordures. Selon lui, cette expérience a été enrichissante du fait qu'elle lui a permis de prendre contact avec des personnes diftérentes de son milieu socio-economique. Aussi, il déclare avoir été valorisé par le fait de rendre service à la communauté. À quelques reprises il a demandé à son fils de 12 ans de l'accompagner au centre dans le but d'améliorer ses relations avec lui.

Pour le superviseur communautaire, sa participation active à une sentence de travaux communautaires lui a permis de modifier la perception négative qu'il avait d'un délinquant. De plus, il se considère satisfait du travail accompli par René... Aussi, il est d'avis que ce type de sentence favorise davantage la réhabilitation d'un individu qu'une période d'emprisonnement. 
Une fois la sentence terminée, il s'est dit disposé à recommencer ce genre d'expérience dans un avenir rapproché.

Le succès de cette sentence incite le juge qui l'avait rendue à recourir de nouveau aux travaux communautaires pour d'autres justiciables.

Satisfait des résultats de cette première expérience, l'agent de probation n'a pas hésité à recommander la même mesure pour d'autres candidats.

\section{b) Deuxième histoire de cas}

Jean... est reconnu coupable par la Cour des Sessions de la Paix de conspiration et de vol qualifié. Le juge demande un rapport présentenciel au service de Probation. Âgé de 18 ans, Jean... est l'aîné d'une famille de deux enfants. Issu d'un milieu bien structuré et sans aucune problématique, il reçoit une bonne éducation lui permettant de vivre adéquatement dans la société d'autant plus que le sujet ne présente aucun trouble majeur de comportement durant son enfance et son adolescence. Au plan scolaire, Jean... complète son secondaire IV, niveau d'instruction qu'il considère comme suffisant pour se lancer sur le marché du travail. Journalier dans une usine, il est perçu comme un bon employé, démontrant une stabilité et une motivation pour le travail.

Depuis un an environ, Jean... fréquente des individus reconnus comme marginaux ou délinquants. Quelques mois avant la perpétration du délit en cause, il est congédié temporairement par son employeur en raison d'une baisse de la production. Oisif, il passe ses journées dans des brasseries ou salles de billard avec ces mêmes amis. Au niveau de la participation au vol qualifié, Jean assume le rôle de conducteur.

Compte tenu de sa participation limitée à cette infraction et considérant le fait qu'il était un individu peu criminalisé, pouvant fonctionner dans la société sans représenter un réel danger pour l'intérêt public, l'agent de probation recommande dans son rapport présentenciel qu'il fasse l'objet d'une sentence de travaux communautaires afin de prendre conscience de ses responsabilités face à la collectivité.

Le juge, après consultation du rapport, donne son accord de principe et demande au service de Probation d'examiner les 
modalités possibles du déroulement d'une sentence de travaux communautaires fixant un quantum de 120 heures.

L'agent de probation entreprend alors des démarches pour contacter un organisme à but non lucratif acceptant la supervision d'une sentence de travaux communautaires et la mise au point d'un horaire conforme aux disponibilités de chacun. Le directeur des services récréatifs de la ville informe l'agent de probation que l'association du baseball mineur a un besoin urgent de bénévoles et le président de cette association est prêt à assumer la supervision de cette sentence au sein de l'organisme. Le travail proposé consiste à devenir aide-entraîneur pour un club de garçons de 12 à 13 ans.

Le jour de la comparution, le juge suspend le prononcé de la sentence pour une période de trois ans et ordonne que Jean... accomplisse une sentence de travaux communautaires d'une durée de 120 heures selon les modalités établies par l'agent de probation. Toutefois, le juge ordonne que le sujet se présente régulièrement au service de Probation durant ces trois années.

Les 35 premières heures de travail communautaire se déroulent sans aucun problème : Jean... respecte son horaire et se sent valorisé par son rôle d'aide-entraîneur. Cependant, la situation se détériore au cours de l'accomplissement des dernières 60 heures. En effet, le superviseur communautaire confie la responsabilité de la surveillance de la sentence à une autre personne, sans aviser au préalable l'agent de probation. De plus, en raison de son manque d'expérience dans le domaine du baseball, on décide d'exclure Jean... comme aide-entraineur sans même en discuter avec l'agent de probation. Il en résulte qu'au terme de la saison de baseball Jean... n'a accompli que 95 des 120 heures prescrites. L'agent de probation doit alors refaire des arrangements avec le directeur des services récréatifs et trouver un organisme de remplacement. Un nouveau horaire est établi et Jean... termine sa sentence.

Cette situation crée certains problèmes pour le justiciable. Il perd toute motivation et une seule chose l'intéresse : accomplir le nombre d'heures exigé par la cour afin de n'avoir aucun problème.

L'évaluation de l'agent de probation est qualifiée de succès théorique, mais le cas a nécessité deux fois plus d'énergie du 
fait que deux ressources communautaires ont dû être contactées. Tirant profit de cette expérience, l'agent de probation devait constater l'importance de bien choisir la ressource devant superviser le justiciable en vue d'assurer un contrôle plus étroit autant vis-à-vis du justiciable que du superviseur communautaire.

\section{c) Troisieme histoire de cas}

Denis... est âgé de 21 ans. Il est second d'une famille de quatre enfants. Connaissant certaines difficultés sur le plan scolaire, il joint le marché du travail à l'âge de 16 ans comme apprenti-menuisier dans la construction et conserve un emploi de façon assez stable malgré le fait qu'il doive travailler pour divers employeurs.

A 18 ans, il commence à éprouver des difficultés de toutes sortes. Handicapé par une mémoire très défaillante dès son jeune âge, il devient agacé par le fait de se faire rappeler constamment le moindre rendez-vous, ou engagement par ses parents et les relations familiales se détériorent. En réaction à cette situation, il emprunte avec le temps certains schèmes de conduite parmi lesquels se retrouvent de nombreux achats d'automobiles, des emprunts inconsidérés, une consommation exagérée de boisson alcoolique qui est la cause d'accidents de voiture, des contraventions et la perte de son permis de conduire. Les reproches parentaux qui s'ensuivent ne font qu'envenimer la situation et Denis... connâ̂t au cours de la même année ses premiers démêlés avec la justice. Il comparaît devant les tribunaux à trois reprises et est reconnu coupable pour des infractions telles que méfait et vols par effraction. Il est d'abord soumis à une ordonnance de probation de deux ans puis fait l'expérience de deux termes d'incarcération.

Au cours de l'été 1977, Denis... doit comparaître à nouveau en cour pour des délits de complot et de vol qualifié perpétré un an et demi plus tôt. La cour demande alors au service de Probation de produire un rapport présentenciel. Du fait que les activités criminelles du candidat ont eu lieu à lintérieur d'une période restreinte de temps remontant à deux ans, considérant qu'il s'est produit une nette amélioration dans le comportement du sujet au niveau financier et occupationnel ainsi qu'au chapitre de ses loisirs, de ses habitudes sociales et de ses relations parentales, l'agent de probation suggère une sentence 
de travaux communautaires comme alternative à l'incarcération. Selon lui, une telle mesure est de nature à faire respecter le principe de rétribution sociale et de permettre au justiciable de pouvoir bénéficier d'un ensemble de faits et circonstances qui lui avaient permis depuis quelque temps d'entreprendre sa remontée sociale.

Le jour du prononcé de la sentence, Denis... est assujetti aux dispositions légales suivantes : sentence suspendue et une ordonnance de probation de deux ans incluant la condition spécifique d'accomplir bénévolement 110 heures de travail communautaire à raison de 12 heures par semaine. Le travail consiste en divers menus travaux de menuiserie pour un centre local de services communautaires de sa région.

Durant le mois qui suit, le procureur de la Couronne en appelle de la sentence en invoquant qu'elle est nettement inadéquate et que le juge de première instance n'a pas tenu compte suffisamment de l'aspect exemplaire et dissuasif que doit comporter une sentence. La cause est entendue en appel six mois plus tard. La décision de la Cour d'Appel dans ce cas revêtait une importance particulière pour l'avenir du projet pilote du fait qu'au moment où la cause était entendue, les 110 heures prescrites avaient déjà été accomplies avec succès par Denis... La cour devait alors non seulement se prononcer sur l'individualité du cas, mais aussi sur la validité d'un tel type de sentence. L'appel est rejeté à l'unanimité par les trois juges et la décision du juge de première instance est maintenue.

Dans son rapport d'évaluation du déroulement de cette sentence tel que remis au juge, l'agent de probation qualifie l'expérience de positive pour l'ensemble des parties, malgré les divers problèmes rencontrés en raison de la nouveauté de cette mesure pénale.

\section{CONCLUSION}

Au terme de cette analyse, il convient de présenter une rétrospective des opinions des différents groupes impliqués dans l'expérience pilote et d'énumérer les principales recommandations du rapport d'évaluation (1978). Nous concluerons en abordant les perspectives d'avenir de cette nouvelle mesure au Québec. 
De façon générale, tous les groupes impliqués de près ou de loin dans le déroulement de l'expérience ont exprimé l'avis que le programme devait être généralisé à tous les districts judiciaires du Québec. La sentence de travaux communautaires est d'abord perçue comme une mesure essentiellement resocialisante, plus humanitaire et moins coûteuse que l'incarcération. Les juges, les procureurs et les agents de probation pour leur part considèrent que c'est en deçà de l'incarcération et au-delà du sursis de sentence que la nouvelle mesure doit figurer dans l'éventail traditionnel des mesures pénales. Ce nouveau type de sentence répond à un besoin, surtout lorsque l'incarcération n'est pas absolument nécessaire. D'ailleurs, plusieurs répondants voient la nécessité de conserver les différents éléments de contrainte reliés à l'exécution de cette sentence comme le bénévolat et l'accomplissement des travaux au cours des heures de loisirs.

De façon plus spécifique, la nouvelle mesure constitue pour les agents de probation un enrichissement dans leur travail en ce sens qu'elle ouvre les portes à une plus grande utilisation des ressources communautaires. En ce qui a trait aux organismes communautaires, plusieurs ont émis l'opinion que le programme leur permettait de participer de façon active à l'administration de la justice et de modifier leur perception erronée de la délinquance. Enfin, en ce qui concerne les justiciables eux-mêmes, plusieurs ont apprécié d'avoir l'occasion de réparer de façon positive le tort criminellement causé et d'être consulté personnellement au niveau du choix de leur sentence.

En août dernier, le rapport final d'évaluation de cette expérience pilote était remis aux autorités du ministère de la Justice. Il était assorti de 28 recommandations relatives à l'application du programme dans tout le Québec, parmi lesquelles on doit citer les suivantes :

- le succès du programme dépend en grande partie d'une banque importante de ressources communautaires variées et fiables ;

- certaines lois déjà existantes au Québec comme celle de la qualification et de la formation professionnelle de la Maind'ceuvre (1969, loi 51, statuts refondus), la Loi de la Commission du salaire minimum (1964, S.R.Q. chap. 144) et la Loi de la Commission des accidents de travail (1964, S.R.Q. chap. 159) doivent être examinées et modifiées s'il y a lieu ; 
- des mécanismes visant à solutionner le problème de la responsabilité en matière civile, concernant les ressources communautaires et les justiciables, doivent être élaborées ;

- la mise en application et le déroulement du programme des travaux communautaires doivent être confiés au service de Probation.

Pour le service de Probation, l'implantation du projet pilote de sentences de travaux communautaires demeure une expérience de travail unique et enrichissante. Les résultats obtenus démontrent que la nouvelle mesure répond à un besoin et qu'elle est applicable sous certaines conditions dans un contexte québécois. Suite à l'étude du rapport d'évaluation présenté au mois d'août 1978, le ministère de la Justice a décidé d'implanter le programme dans tous les districts judiciaires du Québec dès le début de l'année 1980. Pour l'instant, le service de Probation est en train de préparer les mécanismes qui vont permettre la mise en application de ce nouveau type de mesure pénale dans tous les tribunaux québécois. 


\section{BIBLIOGRAPHIE}

BEHA, J., C. KENNETH and R.H. ROSENBLUM (1977); Sentencing to Community Service, U.S. Government Printing Office, Washington, D.C.

COMMISSION DE REFORME DU DROIT DU CANADA (1976) : Le participation communautaire da la rédaptation du délinquant, $\mathrm{O}$ tawa, Approvisionnement et Service, Canada.

GREAT-BRITAIN (1970) : Non-custodial and Semi-custodial Penalties $\gg$, Report of the Advisory Council on the Penal System, London.

INNER LONDON PROBATION AND AFTER-CARE SERVICE (1973) : Community Service by Offender - An Interim Report, September.

MINISTERE DE LA JUSTICE - DIRECTION DE LA PROBATION (1978) : Une expérience pilote de travaux communautaires au Québec - rapport d'évaluation, août, Québec.

NACRO AND HOWARD LEAGUE (1974) : Community Service by Offenders : The Nottinghamshire Experiment, edited by John Harding, London. 